Beckensonografie in einer einzigen Schallebene wurden das Volumen der Ovarien sowie die maximale Follikelzahl bestimmt. Um die jeweiligen altersabhängigen Werte mit der besten Sensitivität und Spezifität zu ermitteln, bedienten sich die Ärzte der ROC-Kurven-Methode (receiver operating characteristic [ROC] curve). Dazu werden die Wertepaare von Spezifität und Sensitivität für alle möglichen Cutoff-Werte in ein Diagramm altersabhängig in Fünf-JahresSchritten notiert. Anhand des YoudenIndex (Sensitivität + Spezifität - 1) ermittelten die Ärzte jeweils den Wert, der für die maximale Trennschärfe stand. Als Schwellenwert verwendeten sie unter anderem für Frauen über 24 ein Volumen von $12 \mathrm{ml}$ und eine Follikelzahl von 13, für Frauen zwischen 25 und 29 ein Volu-

\title{
Postmenopause: ein bisschen besserer Sex dank transdermalem Östrogen
}

\author{
Östrogene, transdermal verabreicht, können dazu beitragen, dass Frauen in \\ der frühen Postmenopause wieder mehr Freude am Sex haben. Der Effekt \\ ist allerdings nur moderat.
}

A nhand des Female Sexual Function Inventory (FSFI) haben Hugh Taylor et al. in einer Studie untersucht, ob sich bei Frauen in der frühen Postmenopause die Sexualfunktion durch den Einsatz von Hormonen verbessern lässt. Der Fragebogen erfasste die Erregung, vaginale Befeuchtung, Orgasmusfähigkeit, $\mathrm{Zu}$ friedenheit und Schmerzen beim Verkehr. Der Wertebereich reicht von 0 bis 36 Punkten (höhere Werte weisen auf eine bessere Sexualfunktion hin). 670 Frauen mit einem Durchschnittsalter von 53 Jahren nahmen teil, die auf 48 Monate angelegt war. 204 von ihnen erhielten im randomisierten und doppelt verblindeten Verfahren transdermales 17-beta-Estradiol $(50 \mu \mathrm{g} / \mathrm{d}), 209$ konjugiertes equines Östrogen oral $(0,45 \mathrm{mg} / \mathrm{d})$ und 257 Placebo, im Fall der Hormongruppen monatlich zwölf Tage lang auch mikronisiertes Progesteron (200 mg).

Signifikante Effekte gegenüber Placebo waren im FSFI-Gesamtscore nur für transdermales Estradiol festzustellen, 30-34-Jährige ein Volumen von $9 \mathrm{ml}$ und eine Follikelzahl von zehn. In einer zweiten Kohortengruppe bestätigte sich nach Angaben der Ärzte, dass für die PCOSDiagnose altersabhängig Volumen- und Follikelzahl gesenkt werden mussten. Die gewählten altersabhängigen CutoffWerte für Ovarvolumen und Follikelzahl lieferten nach Berechnungen der Ärzte eine gute Spezifität und Sensitivität in der Gruppe der Frauen im Alter zwischen 30 und 40 Jahren. Bei jüngeren Frauen sei es mithilfe der gewählten Parameter weniger zuverlässig gelungen, ein PCOS zu entdecken.

Peter Leiner

Kim H-J et al. Polycystic ovary morphology: age-based ultrasound criteria. Fertility and Sterility 2017; online 12. August ; https://doi. org/10.1016/j.fertnstert.2017.07.005 men von $10 \mathrm{ml}$ sowie 14 Follikel, für

\section{Hier steht eine Anzeige.}

Springer

und auch nur bei Frauen mit zu Beginn geringer Sexualfunktion (definiert als FSFI-Score $\leq 26,55$ Punkte). Diese stellten allerdings mit mehr als $70 \%$ in allen Gruppen die Mehrheit. Der mittlere Zugewinn beim FSFI-Wert betrug über den Studienverlauf unter transdermalem Estradiol 3,9 Punkte; statt $74 \%$ wie zu Studienbeginn hatten schließlich nur noch $67 \%$ eine FSFI-Punktzahl unterhalb der Schwelle zur schwachen Sexualfunktion. Betrachtete man die einzelnen Punkte, so führte transdermales Estradiol zu einem Anstieg der vaginalen Befeuchtung $(0,61 ; 95 \%$-Konfidenzintervall [95\%-KI]: $0,25-0,97 ; p=0,001)$ und zu verminderten Schmerzen (0,67; $95 \%$-KI: 0,25-1.09; $\mathrm{p}=0,002)$ im Vergleich zu Placebo.

\section{Dr. Robert Bublak}

Taylor HS et al. Effects of Oral vs Transdermal Estrogen Therapy on Sexual Function in Early Postmenopause Ancillary Study of the Kronos Early Estrogen Prevention Study (KEEPS). JAMA Internal Med. 2017, online 28. August; https:// doi.org/10.1001/jamainternmed.2017.3877 